\title{
Design and Analysis of Self-excited Miniature Magnetic
}

\section{Generator}

\author{
Wu-Sung Yao and Wei-Quan Jian \\ Department of Mechanical and Automation Engineering, National Kaohsiung First University of Science and Technology, \\ Kaohsiung 824, Taiwan
}

Received: March 06, 2015 / Accepted: March 20, 2015 / Published: March 25, 2015.

\begin{abstract}
Nowadays, electronic devices are more and more integrated into everyday life. These seamless integrations focus on mobility, but at the same time strive to be unobtrusive to the end user. With the introduction of personal data assistants and intelligent cellular phones for the searching of the website, true mobile computing is closer than ever. However, battery technology, which powers most of these mobile connectivity solutions, has not kept up the same pace of improvement. The paper describes a methodology for the design and performance of a self-excited permanent-magnet generator applied to low power supplies. It combines an analytical field model, a lumped reluctance equivalent magnetic circuit, and an equivalent electrical circuit. An illustrated example of a 15-mW, 290-r/min generator is given, and the analysis techniques are validated by measurements on a prototype system.
\end{abstract}

Key words: Permanent-magnet generator, analytical field model, equivalent magnetic circuit.

\section{Introduction}

With the proliferation of portable electronic consumer products and electronic security devices, there is an increasing need for low power supplies. In many applications, on-board power generation for the portable electronic products would be preferable to the use of batteries with limited capacity and lifetime. By incorporating a miniature permanent-magnet generator, generating electrical power is to directly convert mechanical energy to electrical energy [1]. A mechanical input power can be derived from desultory movements, which might be associated with the stochastic motion of a limb, such as the arm, or due to a specific action, such as cellular phone sharking [2, 3, 5]. Permanent-magnet generators systems which are capable of extracting and storing energy from both reciprocating and intermittent motion have been reported previously. However, in common with many

Corresponding author: Wu-Sung Yao, Ph.D., research fields: applications of servomotor control, learning control theory, and synchronization control. E-mail: wsyao@nkfust.edu.tw. other direct-drive electromagnetic devices, these tend to have a relatively poor specific power capability since the input speed is limited. Hence, rather than directly converting the kinetic energy to electrical energy, it is advantageous using efficiency and specific power capability to initially accumulate the mechanical input energy. An approach is employed in kinetic "self-excited" method, which utilizes kinetic energy associated with wrist movements [6, 7].

Magnetic generator can provide a next level of performance required for higher endurance, higher energy saving and reduced charging times when $3 \mathrm{C}$ products were run out of battery. Although they will not totally displace conventional charging system for many applications, they provide a practical solution for inconvenience from portable battery $[4,8]$. The traditional bearings are widely used in conventional, but may take many friction consumption in machines, most of the energy consumed by friction and reduce machine efficiency. New technology is required to push the limits even further and achieve a new standard of capability in performance and throughout. In this 
paper, a methodology for the design and performance of a self-excited permanent-magnet generator is described. It combines an analytical field model, a lumped reluctance equivalent magnetic circuit, and an equivalent electrical circuit. A magnetic generator with magnetic bearings is proposed. Using magnetic bearings element eliminates most of these consumption. The magnetic bearings consist of many non-contact elements. With the magnetic generator, the magnetic force directly engages the rotating with no mechanical connection. There is no mechanical friction or concussion. Rotor of magnetic generator is forced by magnetic force attract and repulse, but rotor may stop because magnetic force of magnets is consumed until exhausted. Magnetic bearings can reduce the rate of consumption effectively, so we use rotating power to generator electricity with toroid coil.

The paper is organized as follows: Section 2 explains the design concept and magnetic field analysis; Section 3 introduces the experimental evaluation; Section 4 presents experimental results and discussions; and Section 5 gives conclusions.

\section{Design and Magnetic Field Analysis}

In this study, the magnetic generator contains several parts like power section, bearing section, coil and electromagnetic section. In power section, the same poles produced a vertical resistance by magnets in left side of Fig. 1. When the magnet can be rotated with an angle, it produced not only a vertical resistance but also a horizontal resistance, in right side of Fig. 1. Therefore, rotor of magnetic generator can be forced by using horizontal resistance as like Fig. 1.

In practical, the degrees of freedom of magnets must be limited. In left side of Fig. 2, it shows that same pole of magnets can produce a vertical resistance in positive direction of y axis and horizontal resistance in positive direction of $\mathrm{x}$ axis. In right side of Fig. 2, it shows that different pole of magnets can produce a vertical resistance in negative direction of $y$ axis and horizontal resistance in positive direction of $\mathrm{x}$ axis. The

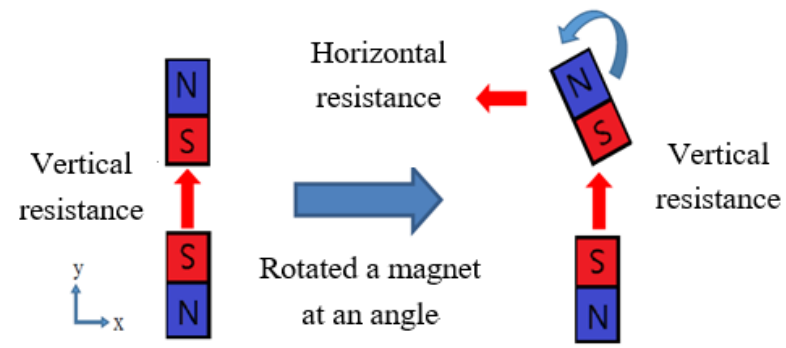

Fig. 1 Source of kinetic by magnets.

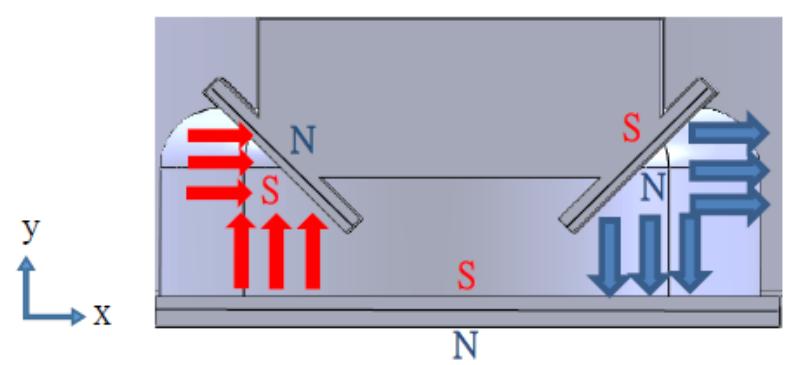

Fig. 2 Result of assumptions.

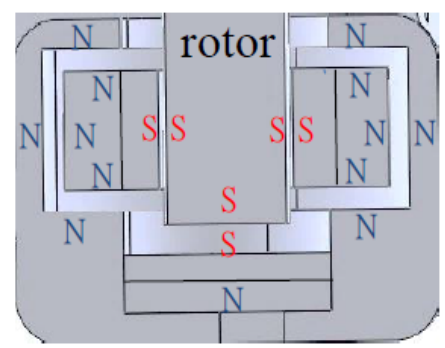

Fig. 3 Distribution of magnetic.

summation of forces in $\mathrm{y}$ directions should be zero; in $\mathrm{x}$ direction must not be zero. In other words, there is a force in positive direction of $x$, and rotor of magnetic generator is rotating by this force for self-excited.

In bearing section, in order to continue kinetic energy from magnets and reduce friction, a magnetic bearing for self-excited miniature magnetic generator can be designed as like Fig. 3. It keeps the rotor in a center position and retaining a margin from boundary, floating the rotor onto air and avoiding collision with other parts. In which the magnetic bearing is using radiation magnetizing, and distribution of poles is belongs to one pole on inner diameter and one pole on outer diameter.

In electromagnetic section, an induced back electromotive force is generated in DC (direct current) brush motor when it is rotating. This back 
electromotive force can also be a source of electricity in magnetic generator. There is a formula regarding to the magnitude of back electromotive force, i.e., $E=\frac{P Z}{60 a} \varphi n$.

In this formula, $P$ represents quantity of magnets, $\mathrm{Z}$ represents quantity of conductors, $a$ represents parallel circuit of brush, $\varphi$ represents magnetic flux, and $n$ represents motor speed. In this formula, the quantity of magnetic poles is proportional to back electromotive force. Therefore, we can increase quantity of magnetic poles in magnetic generator to produce more electricity as shown in Fig. 4.

In coil section, electricity can be produced for magnets having a relative movement with coil, and produce a resistance to the movement of magnets or coils.

In order to eliminate this resistance, a toroid coil (as shown in Fig. 6) can be given which could not produce a resistance to external magnetic field, because it is circularity and it can generate a closed-loop of magnetic field in the interior of toroid coil. Fig. 6 shows a picture of toroid coil and magnetic field which can be produced in toroid coil. The prototype of magnetic generator is shown in Fig. 7.

\section{Experimental Evaluation}

Fig. 8 shows the experimental setup for the power section, used a general bearing replaced magnetic bearings to keep the rotor in a center position. The magnets are located at a specific position in acrylics, and adjustable height by screw just for generator maximum power.

The proposed power section is shown in Fig. 2. Supposed that there is a summation of forces in $\mathrm{x}$

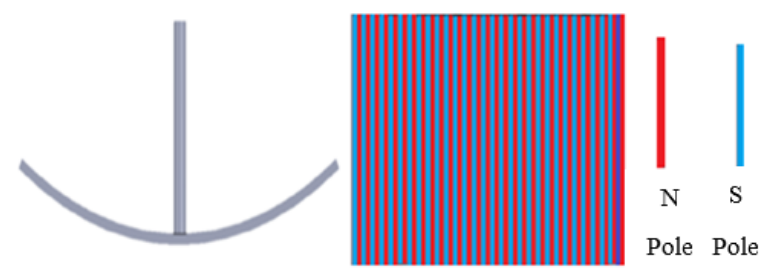

Fig. 4 Magnetizing in multipoles.

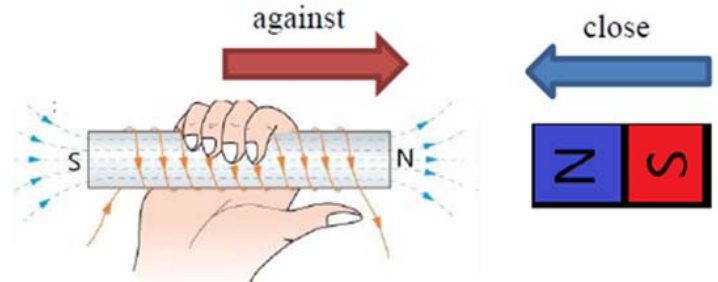

Fig. 5 Phenomenon of electromagnetic induction.

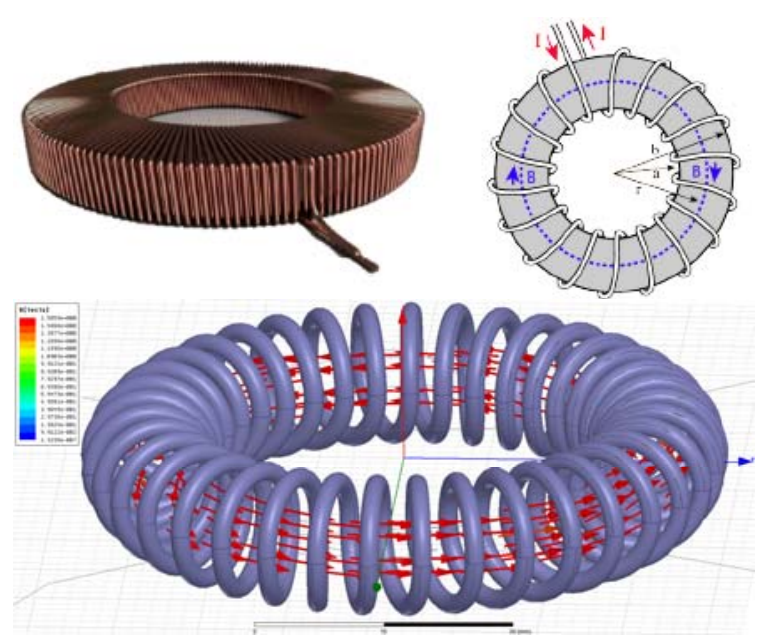

Fig. 6 Toroid coil and magnetic field in toroid coil.

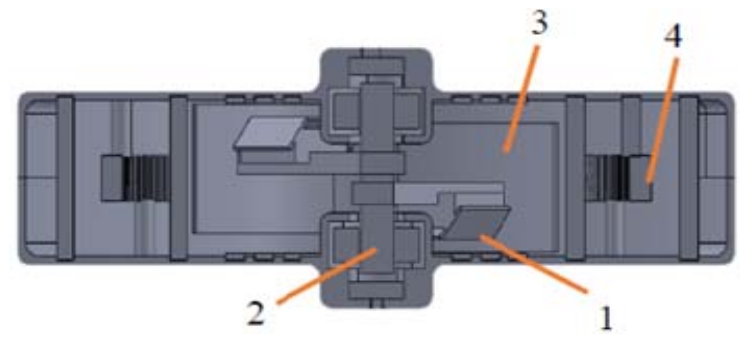

Fig. 7 Prototype of magnetic generator: (1) power section; (2) bearing section; (3) electromagnetic section; (4) coil section.

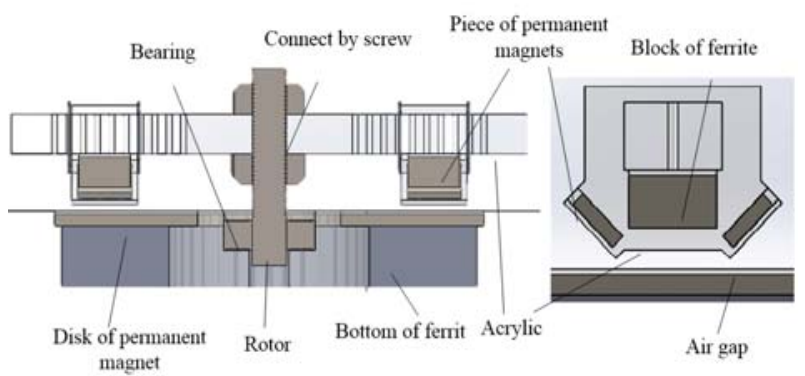

Fig. 8 Experiment setup.

direction pushes objects to go forward. But in more powerful evidence, the magnetic flux bypasses can be realized with magnets closing to the same poles; while the magnetic flux passes with magnets closing to the different poles as shown in Fig. 9. 
Table 1 Parameter of experiment.

\begin{tabular}{llll}
\hline & Dimension & $\begin{array}{l}\mathrm{Br} \\
(\text { Gauss })\end{array}$ & $\begin{array}{l}\mathrm{Hc} \\
(\mathrm{KA} / \mathrm{m})\end{array}$ \\
\hline $\begin{array}{l}\text { Piece of } \\
\text { permanent }\end{array}$ & $18.8 \times 11.5 \times 3 \mathrm{mmT}$ & 11,700 & 955 \\
$\begin{array}{l}\text { magnets } \\
\begin{array}{l}\text { Disk of permanent } \\
\text { magnets }\end{array}\end{array}$ & $\mathrm{OD} 150 \times \mathrm{ID} 50 \times 4$ & 11,700 & 955 \\
$\begin{array}{l}\text { Block of ferrite } \\
\text { Bottom of ferrite }\end{array}$ & $\begin{array}{l}18 \times 18 \times 10 \mathrm{mmT} \\
\mathrm{OD} 145 \times \mathrm{ID} 70 \times 20\end{array}$ & 3,800 & 161 \\
Air gap & $3 \mathrm{~mm}$ & 3,800 & 161 \\
\hline
\end{tabular}

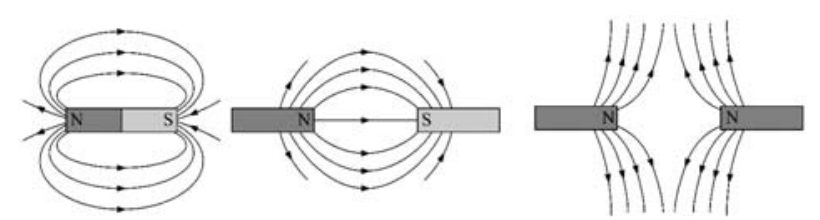

Fig. 9 Phenomenon of magnetic flux.

In this illustrated example, a geometric model of power section is obtained using magnetic analysis software. First, the predicted flux distribution can be verified. The magnetic flux in left side of Fig. 10 is used to produce a trend of bypassed, and magnetic flux in right side of Fig. 10 is used to produce a trend of passes. But choice of conductor materials is also an important factor, material with a high permeability increasing magnetic flux, but eddy current can be found in conductor which is a resistance to the magnetic flux flow through conductor. There is a material called ferrite which has magnetic conductibility
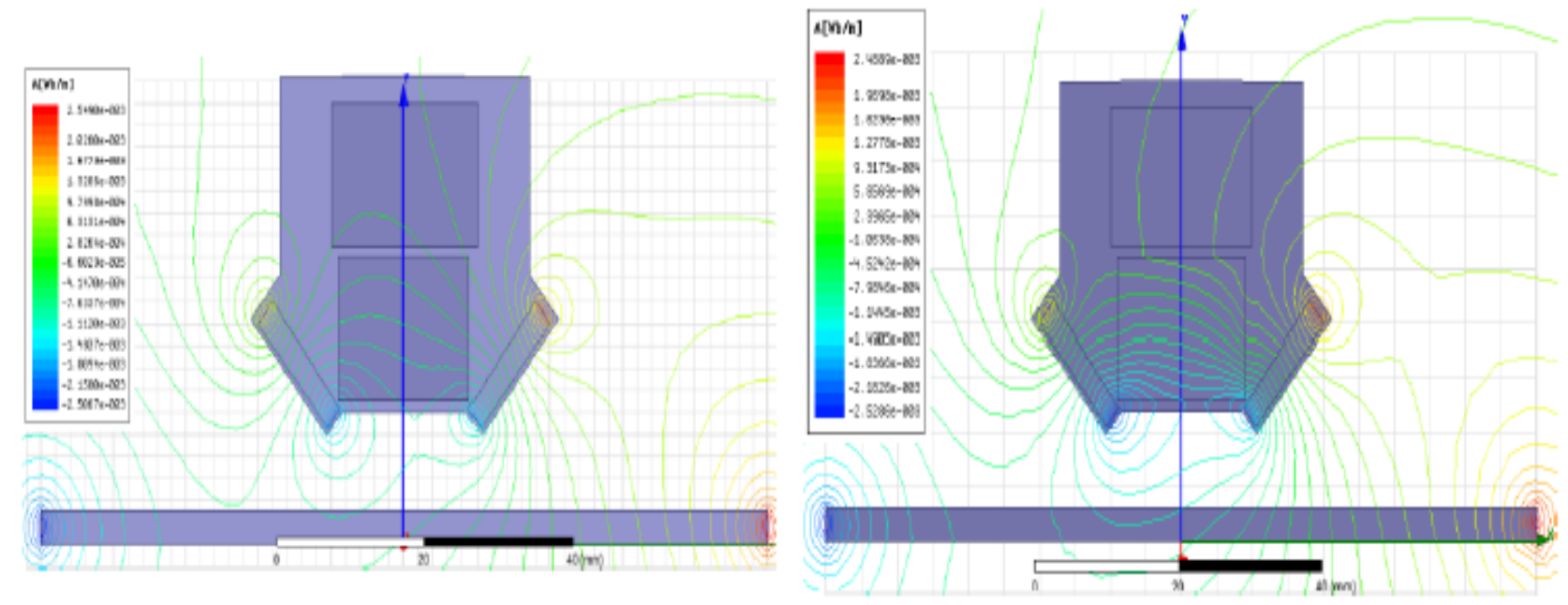

but non-conductive and put this material in acrylic between magnets of left side and right side. Fig. 10 shows a significant difference regarding ferrite presences or absences, and situation about the magnetic flux.

An experiment setup is constructed and used to verify the conception of the proposed power section, which is feasible and increasing magnetic flux in used of conductor of ferrite. It bring an advantage is about the acceleration and coercive force are increased, extend the use time of the magnets, and could not produce an eddy current break.

\section{Experimental Results}

The proposed generator having the design parameters in Table 1 is shown in Fig. 11. There is an accurate prediction to illustrate the experiment setup being feasible, and generate an acceleration to rotate for rotor by magnetic force. At 20 s of Fig. 12a, the acceleration is balanced by friction and winding, and rotor is keeping the same speed of $290 \mathrm{rpm}$. In Fig. 12b, the steady state of the position response can be found after 20 s. In Fig. 12c, as speed increases, the amplitude of torque is increasing and the measured maximum of the torque is $126 \mathrm{mNm}$.

\section{Conclusions}

The paper describes a methodology for the design

Fig. 10 Predicted flux distribution. 


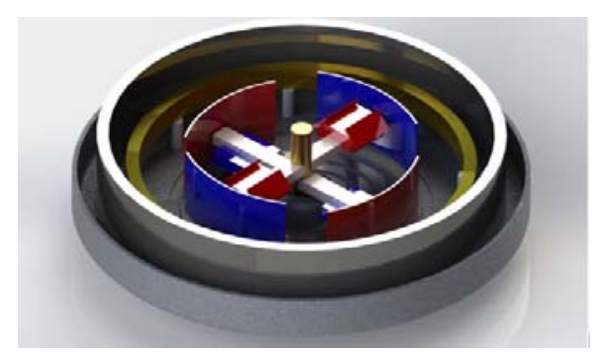

Fig. 11 Prototype of the generator.

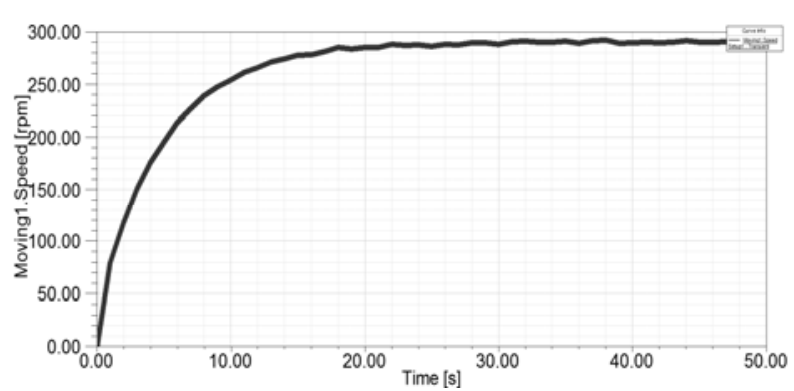

(a)

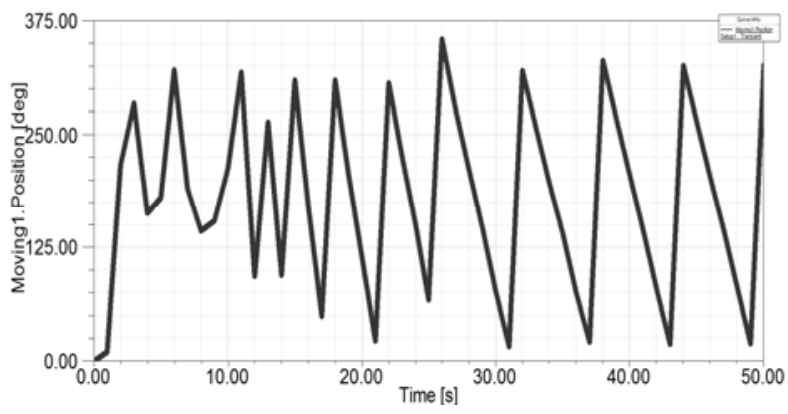

(b)

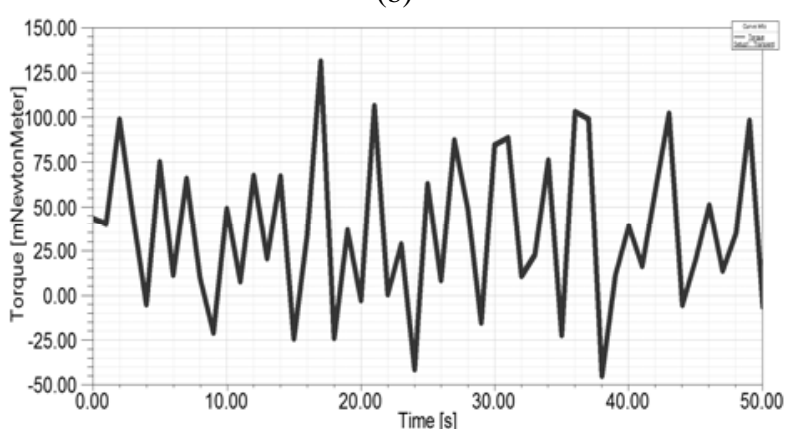

(c)

Fig. 12 Experimental results: (a) time response of the generator's speed; (b) time response of the generator's position and; (c) time response of the generator's torque. and performance of a self-excited permanent-magnet generator applied to low power supplies. It combines an analytical field model, a lumped reluctance equivalent magnetic circuit, and an equivalent electrical circuit. An illustrated example of a $15-\mathrm{mW}$, $290-\mathrm{r} / \mathrm{min}$ generator is analyzed and validated by measurements on a prototype system.

\section{References}

[1] Williams, C. B., and Yates, R. B. 1996. "Analysis of a Micro-electric Generator for Microsystems.” Sensors and Actuators A-physical 52 (1): 8-11.

[2] Wang, J., Wang, W., Jewell, G. W., and Howe, D. 1998. "Design and Experimental Characterization of a Linear Reciprocating Generator.” In Proc. IEE-Electric Power Application, 509-18.

[3] Wang, J., Wang, W., Jewell, G. W., and Howe, D. 1998. "Analysis and Design of a Linear Reciprocating Generator for Telemetry-Based Vibration Monitoring System.” In Proc. ICEM'98, 857-62.

[4] Wang, J., Wang, W., Jewell, G. W., and Howe, D. 2002. "A Low-Power, Linear, Permanent-Magnet Generator/Energy Storage System.” IEEE Transactions on Industrial Electronic 49 (3): 640-8.

[5] Galchev, T., Kim, H., and Najafi, K. 2011. "Micro Power Generator for Harvesting Low-Frequency and Nonperiodic Vibrations.” Journal of Microelectromechanical Systems 20 (4): 852-661.

[6] Tabesh, A., and Frechette, L. G. 2010. “A Low-Power Stand-Alone Adaptive Circuit for Harvesting Energy from a Piezoelectric Micropower Generator.” IEEE Transactions on Industrial Electronic 57 (3): 840-9.

[7] Leijon, M., Bernhoff, H., Ågren, O., Isberg, J., Sundberg, J., Berg, M., Karlsson, K. E., and Wolfbrandt, A. 2005. "Multiphysics Simulation of Wave Energy to Electric Energy Conversion by Permanent Magnet Linear Generator.” IEEE Transactions on Energy Conversion 20 (1): 219-24.

[8] Rankin, D. D., Leal, E. N. M., and Walther, D. C. 2005. "Personal Power Systems.” Prog. Energy Combust. Sci. 31: 422-65. 